\title{
SEQUENCES OF BOUNDS FOR THE SPECTRAL RADIUS OF A POSITIVE OPERATOR
}

\author{
ROMAN DRNOVŠEK
}

\begin{abstract}
In 1992, Szyld provided a sequence of lower bounds for the spectral radius of a nonnegative matrix $A$, based on the geometric symmetrization of powers of $A$. In 1998, Taşçi and Kirkland proved a companion result by giving a sequence of upper bounds for the spectral radius of $A$, based on the arithmetic symmetrization of powers of $A$. In this note, we extend both results to positive operators on $L^{2}$-spaces.
\end{abstract}

Key words: numerical radius, spectral radius, positive operators, kernel operators Math. Subj. Classification (2010): 47A12, 47A10, 47B34, 47B60

\section{INTRODUCTION}

Let $A=\left(a_{i j}\right)_{i, j=1}^{n}$ be a nonnegative matrix, i.e., $a_{i, j} \geq 0$ for all $i$ and $j$. In the literature, much attention has been paid to provide upper and lower bounds for the spectral radius $r(A)$ of $A$. Here $r(A)$ is defined as $\max \left\{\left|\lambda_{1}\right|, \ldots,\left|\lambda_{n}\right|\right\}$, where $\left\{\lambda_{i}\right\}_{i=1}^{n}$ are the eigenvalues of $A$. This number is an eigenvalue of $A$, and it is called the Perron root of $A$. Szyld [5] gave an increasing sequence of lower bounds for $r(A)$ that are based on the geometric symmetrization of powers of $A$. More precisely, let $S(A)$ be the matrix whose $(i, j)$ entry is equal to $\sqrt{a_{i j} a_{j i}}$, and define $\rho_{k}=r\left(S\left(A^{2^{k}}\right)\right)^{2^{-k}}$. It is shown in [5] that $\rho_{0} \leq \rho_{1} \leq \ldots \leq \rho_{k} \leq r(A)$ for all $k$. Easy examples show that the sequence $\left\{\rho_{k}\right\}_{k \in \mathbb{N}}$ does not converge to $r(A)$ in general; if we take e.g.

$$
A=\left(\begin{array}{lll}
0 & 1 & 0 \\
0 & 0 & 1 \\
1 & 0 & 0
\end{array}\right)
$$

then $\rho_{0}=\rho_{1}=\ldots=0$, while $r(A)=1$. On the other hand, Taşçi and Kirkland [6] provided a decreasing sequence of upper bounds for $r(A)$ that are based on the arithmetic symmetrization of powers of $A$. Specifically, let $M(A)=\left(A+A^{T}\right) / 2$ and define $\sigma_{k}=$ $r\left(M\left(A^{2^{k}}\right)\right)^{2^{-k}}$. It is proved in [6] that $\sigma_{0} \geq \sigma_{1} \geq \ldots \geq \sigma_{k} \geq r(A)$ for all $k$ and that the sequence $\left\{\sigma_{k}\right\}_{k \in \mathbb{N}}$ converges to $r(A)$. In this paper we extend both results to positive operators on $L^{2}$-spaces. We should mention that in [1] the inequality $\sigma_{0}=r(M(A)) \geq$ $r(A)$ was already extended to this setting.

LINEAR ALGEBRA AND ITS APPLICATIONS 574 (2019), 40-45 
Throughout the note, let $\mu$ be a $\sigma$-finite positive measure on a set $X$. We consider bounded (linear) operators on the complex Hilbert space $L^{2}(X, \mu)$. The norm in $L^{2}(X, \mu)$ is denoted by $\|\cdot\|_{2}$. An operator $A$ on $L^{2}(X, \mu)$ is said to be positive if it maps nonnegative functions to nonnegative ones. Given operators $A$ and $B$ on $L^{2}(X, \mu)$, we write $A \geq B$ if the operator $A-B$ is positive. The operator norm and the spectral radius of an operator are denoted by $\|\cdot\|$ and $r(\cdot)$, respectively. The numerical radius of an operator $A$ on $L^{2}(X, \mu)$ is defined by

$$
w(A):=\sup \left\{|\langle A f, f\rangle|: f \in L^{2}(X, \mu),\|f\|_{2}=1\right\} .
$$

If, in addition, $A$ is positive, then we have

$$
w(A)=\sup \left\{\langle A f, f\rangle: f \in L^{2}(X, \mu), f \geq 0,\|f\|_{2}=1\right\}
$$

Indeed, this follows from the estimate

$$
|\langle A f, f\rangle| \leq \int_{X}|A f||f| d \mu \leq\langle A|f|,|f|\rangle
$$

that holds for any $f \in L^{2}(X, \mu)$. It is well-known [4] that

$$
r(A) \leq w(A) \leq\|A\|
$$

for all bounded operators $A$ on $L^{2}(X, \mu)$. If, in particular, $A$ is selfadjoint, then we have $r(A)=w(A)=\|A\|$.

Let $A$ be a positive operator on $L^{2}(X, \mu)$. The arithmetic symmetrization $M(A)$ of $A$ is the positive selfadjoint operator on $L^{2}(X, \mu)$ defined by $M(A)=\left(A+A^{*}\right) / 2$. Since $\langle M(A) f, f\rangle=\langle A f, f\rangle$ for any nonnegative function $f \in L^{2}(X, \mu)$, we have

$$
w(M(A))=w(A)
$$

Let $K$ be a positive kernel operator on $L^{2}(X, \mu)$ with a kernel $k$, that is, $k: X \times$ $X \rightarrow[0, \infty)$ is a measurable function such that $(K f)(x)=\int_{X} k(x, y) f(y) d \mu(y)$ for all $f \in L^{2}(X, \mu)$ and for almost all $x \in X$. The geometric symmetrization $S(K)$ of $K$ is the positive selfadjoint kernel operator on $L^{2}(X, \mu)$ with the kernel equal to $\sqrt{k(x, y) k(y, x)}$ at a point $(x, y) \in X \times X$. Note that $S(K)$ is well-defined on the whole $L^{2}(X, \mu)$, because the kernel of $S(K)$ is smaller than or equal to the kernel of $M(K)$ by the inequality of arithmetic and geometric means. It was proved in [2, Proposition 2.7] that

$$
r(S(K)) \leq r(K)
$$

Note that our results do not generalize beyond $L^{2}$-spaces. 


\section{Results}

We begin with an observation that seems to be new also in the finite-dimensional case.

Lemma 2.1. If $K$ is a positive kernel operator on $L^{2}(X, \mu)$, then

$$
S\left(K^{2}\right) \geq S(K)^{2} \text {. }
$$

Proof. Let us compare the kernels of both operators. If $k$ is the kernel of $K$, then the kernel of $K^{2}$ at a point $(x, y) \in X \times X$ is equal to $\int_{X} k(x, z) k(z, y) d \mu(z)$, and so the kernel of $S\left(K^{2}\right)$ at a point $(x, y)$ equals

$$
\sqrt{\left(\int_{X} k(x, z) k(z, y) d \mu(z)\right)\left(\int_{X} k(y, z) k(z, x) d \mu(z)\right)} .
$$

On the other hand, the kernel of $S(K)^{2}$ at a point $(x, y)$ is equal to

$$
\int_{X} \sqrt{k(x, z) k(z, x)} \sqrt{k(z, y) k(y, z)} d \mu(z) .
$$

Now the desired inequality is proved by an application of the Cauchy-Schwarz inequality.

We now extend the finite-dimensional result due to Szyld [5, Theorem 2.2].

Theorem 2.2. Let $K$ be a positive kernel operator on $L^{2}(X, \mu)$, and let $\rho_{n}=r\left(S\left(K^{2^{n}}\right)\right)^{2^{-n}}$, $n \in \mathbb{N} \cup\{0\}$. Then, for each $n$,

$$
\rho_{0} \leq \rho_{1} \leq \ldots \leq \rho_{n} \leq r(K) .
$$

Proof. By (2), we have $r\left(S\left(K^{2^{n}}\right)\right) \leq r\left(K^{2^{n}}\right)=r(K)^{2^{n}}$, which implies that $\rho_{n} \leq r(K)$ for all $n \in \mathbb{N} \cup\{0\}$. To finish the proof, it is enough to show that

$$
r\left(S\left(K^{2}\right)\right) \geq r(S(K))^{2} .
$$

By Lemma2.1, we have $\left.S\left(K^{2}\right)\right) \geq S(K)^{2}$ which implies easily that $r\left(S\left(K^{2}\right)\right) \geq r\left(S(K)^{2}\right)=$ $r(S(K))^{2}$ as desired.

The following theorem is an infinite-dimensional generalization of [6, Theorems 1 and 2].

Theorem 2.3. Let $A$ be a positive operator on $L^{2}(X, \mu)$, and let $\sigma_{n}=r\left(M\left(A^{2^{n}}\right)\right)^{2^{-n}}$, $n \in \mathbb{N} \cup\{0\}$. Then, for each $n$,

$$
\sigma_{0} \geq \sigma_{1} \geq \ldots \geq \sigma_{n} \geq r(A) .
$$

Furthermore, the sequence $\left\{\sigma_{n}\right\}_{n \in \mathbb{N}}$ converges to $r(A)$. 
Proof. By (11), we have

$$
r\left(M\left(A^{2^{n}}\right)\right)=w\left(M\left(A^{2^{n}}\right)\right)=w\left(A^{2^{n}}\right) \geq r\left(A^{2^{n}}\right)=r(A)^{2^{n}},
$$

and so $\sigma_{n} \geq r(A)$ for all $n$.

To show that the sequence $\left\{\sigma_{n}\right\}_{n \in \mathbb{N}}$ is decreasing, it is enough to see that $r\left(M\left(A^{2}\right)\right) \leq$ $r(M(A))^{2}=r\left(M(A)^{2}\right)$, or equivalently $w\left(M\left(A^{2}\right)\right) \leq w\left(M(A)^{2}\right)$. It follows from the inequality $\left\|A f-A^{*} f\right\|_{2}^{2} \geq 0$ that we have

$$
\left\langle A^{*} A f, f\right\rangle+\left\langle A A^{*} f, f\right\rangle \geq 2\left\langle A^{2} f, f\right\rangle
$$

for all nonnegative functions $f \in L^{2}(X, \mu)$. This implies that

$$
\left\langle\left(A+A^{*}\right)^{2} f, f\right\rangle \geq 4\left\langle A^{2} f, f\right\rangle
$$

and so we obtain the desired inequality $w\left(M(A)^{2}\right) \geq w\left(A^{2}\right)=w\left(M\left(A^{2}\right)\right)$, where we have also used (1).

For each $n \in \mathbb{N} \cup\{0\}$ we have

$$
r\left(M\left(A^{2^{n}}\right)\right)=w\left(M\left(A^{2^{n}}\right)\right)=w\left(A^{2^{n}}\right) \leq\left\|A^{2^{n}}\right\|,
$$

and so

$$
r(A) \leq \sigma_{n} \leq\left\|A^{2^{n}}\right\|^{2^{-n}}
$$

Since $\left\|A^{2^{n}}\right\|^{2^{-n}} \rightarrow r(A)$ as $n \rightarrow \infty$, the sequence $\left\{\sigma_{n}\right\}_{n \in \mathbb{N}}$ converges to $r(A)$. This completes the proof.

Examples in [6] explain Theorem 2.3 in the finite-dimensional case. The following example further illustrates it in the infinite-dimensional setting.

Example 2.4. Let $A$ be a weighted unilateral shift on $l^{2}$ with weights $\{1,4,1,4,1,4, \ldots\}$, that is, the operator defined by $A\left(x_{1}, x_{2}, \ldots\right)=\left(0, x_{1}, 4 x_{2}, x_{3}, 4 x_{4}, \ldots\right)$. Then $M(A) x=$ $\frac{1}{2}\left(x_{2}, x_{1}+4 x_{3}, 4 x_{2}+x_{4}, x_{3}+4 x_{5}, 4 x_{4}+x_{6}, \ldots\right)$. It is not difficult to verify that $r(M(A))=$ $\|M(A)\|=5 / 2$, and so $\sigma_{0}=5 / 2$. Furthermore, $A^{2} x=\left(0,0,4 x_{1}, 4 x_{2}, 4 x_{3}, \ldots\right)=4 S^{2} x$, where $S$ is the unilateral shift on $l^{2}$. Therefore, $r(A)^{2}=r\left(A^{2}\right)=4 r(S)^{2}=4$, and so $r(A)=2$. Since $M\left(A^{2}\right)=2\left(S^{2}+\left(S^{*}\right)^{2}\right)$, we have $r\left(M\left(A^{2}\right)\right)=\left\|M\left(A^{2}\right)\right\|=4$, so that $\sigma_{1}=2$. Similarly, we obtain that $\sigma_{n}=2$ for all $n=2,3,4, \ldots$

More generally, let $p$ be a positive integer, and let $A_{p}$ be a weighted unilateral shift on $l^{2}$ with weights $\{\underbrace{1,1, \ldots, 1}_{2^{p}-1}, 2^{2^{p}}, \underbrace{1,1, \ldots, 1}_{2^{p}-1}, 2^{2^{p}}, \ldots\}$, so that $A_{1}=A$. Then $A_{p}^{2^{p}}=2^{2^{p}} S^{2^{p}}$, and $r\left(A_{p}\right)^{2^{p}}=r\left(A_{p}^{2^{p}}\right)=2^{2^{p}} r(S)^{2^{p}}=2^{2^{p}}$, so that $r\left(A_{p}\right)=2$. Since $M\left(A_{p}^{2^{p}}\right)=2^{2^{p}-1}\left(S^{2^{p}}+\right.$ $\left.\left(S^{*}\right)^{2^{p}}\right)$, we have $r\left(M\left(A_{p}^{2^{p}}\right)\right)=\left\|M\left(A_{p}^{2^{p}}\right)\right\|=2^{2^{p}}$, so that $\sigma_{p}=2$. Then $\sigma_{n}=2$ for all $n \geq p$ by Theorem 2.3. One can also show that $\sigma_{0}=r\left(M\left(A_{p}\right)\right)=\left\|M\left(A_{p}\right)\right\| \geq 2^{2^{p}-1}$ and that $\sigma_{n}>2$ for all $n<p$. 
We complete this note with an application of the inequality (2). Several authors have studied the spectrum and the spectral radius of selfadjoint kernel operators. If $K$ is a positive kernel operator on $L^{2}(X, \mu)$, then $S(K)$ is a selfadjoint kernel operator. Therefore, if we can compute $r(S(K))$, then the inequality (2) provides the lower bound for $r(K)$. Let us illustrate this with the following proposition.

Proposition 2.5. Let $g:[0,1] \times[0,1] \rightarrow[0, \infty)$ be a measurable function such that, for some $M \geq 1, \frac{1}{M} \leq g(x, y) \leq M$ and $g(x, y) g(y, x)=1$ for all $x$ and $y$. Then the function $k(x, y)=\min \{x, y\} \cdot g(x, y)$ is the kernel of the positive kernel operator $K$ on $L^{2}([0,1])$, and we have

$$
r(K) \geq \frac{4}{\pi^{2}}
$$

Proof. Since $k(x, y)$ is a bounded nonnegative function, it defines the positive kernel operator $K$ on $L^{2}([0,1])$. The kernel of $S(K)$ at a point $(x, y) \in[0,1] \times[0,1]$ is equal to $\min \{x, y\}$. It follows from [3], Exercise 6.5.3, p.103 and p.271] that $r(S(K))=4 / \pi^{2}$. Now, the proof is finished with an application of the inequality (2).

Acknowledgment. The author acknowledges the financial support from the Slovenian Research Agency (research core funding No. P1-0222).

\section{REFERENCES}

[1] R. Drnovšek, A generalization of Levinger's theorem to positive kernel operators. Glasg. Math. J. 45 (2003), no. 3, 545-555.

[2] R. Drnovšek, A. Peperko, Inequalities for the Hadamard weighted geometric mean of positive kernel operators on Banach function spaces, Positivity 10 (2006), no. 4, 613-626.

[3] Y. Eidelman, V. Milman, A. Tsolomitis, Functional analysis. An introduction, Graduate Studies in Mathematics 66, American Mathematical Society, Providence, 2004.

[4] K.E. Gustafson, D.K.M. Rao, Numerical range. The field of values of linear operators and matrices, Universitext, Springer-Verlag, New York, 1997.

[5] D. B. Szyld, A sequence of lower bounds for the spectral radius of nonnegative matrices, Linear Algebra Appl. 174 (1992), 239-242.

[6] D. Taşçi, S. Kirkland, A sequence of upper bounds for the Perron root of a nonnegative matrix, Linear Algebra Appl. 273 (1998), 23-28.

Roman Drnovšek

Department of Mathematics

Faculty of Mathematics and Physics

University of Ljubljana

Jadranska 19

SI-1000 Ljubljana, Slovenia

e-mail : roman.drnovsek@fmf.uni-lj.si 